\title{
Érosion et accrétion de matériaux granulaires humides
}

Alban Sauret $^{(1,2)}$, Guillaume Saingier ${ }^{(1)}$ et Pierre Jop ${ }^{(1)}$ (pierre.jop@saint-gobain.com)

(1) Surface du Verre et Interfaces (UMR125 CNRS/Saint-Gobain), 93303 Aubervilliers Cedex

(2) Department of Mechanical Engineering, University of California,

Santa Barbara, CA 93106, USA

\section{Le comportement des matériaux}

granulaires humides hétérogènes,

\section{éléments incontournables}

de nombreux processus industriels

et géophysiques, résiste encore

à une modélisation complète.

\section{L'interaction des grains avec}

une phase liquide doit être

éclaircie afin d'optimiser

les procédés de mélange,

notamment la dispersion

des grains d'un agrégat cohésif.

Modéliser ces interactions

permettrait aussi de mieux décrire des éboulements granulaires

dont les propriétés mécaniques

évoluent avec des variations

de teneur en liquide

et de répartition spatiale.

\section{Des recherches actuelles}

se tournent vers la compréhension

des échanges dans ces matériaux,

pour isoler les mécanismes

par lesquels des grains

se mélangent à un liquide

ou à une phase granulaire humide.

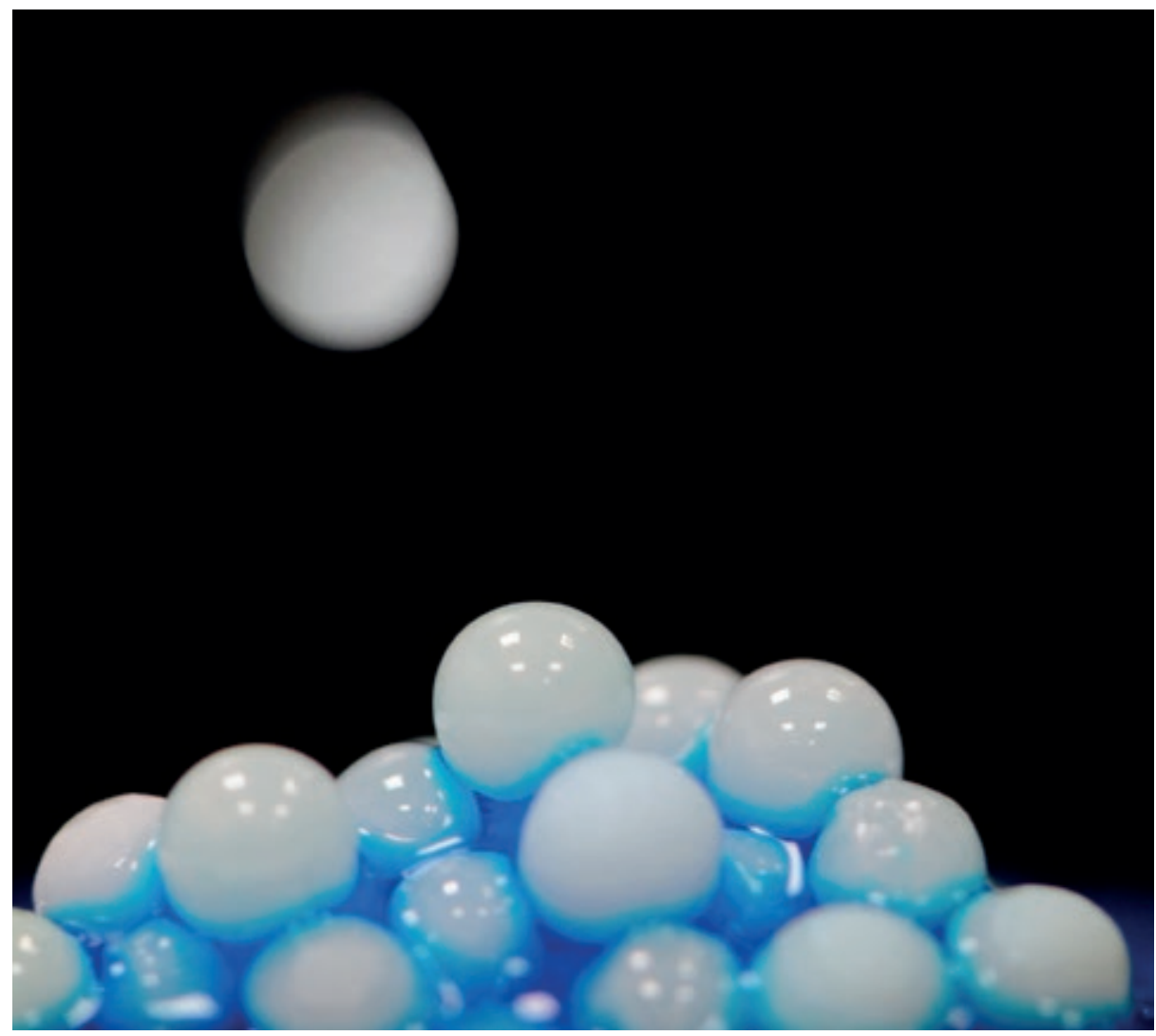

1. Exemple de milieu granulaire (billes de sucre de $3,8 \mathrm{~mm}$ de diamètre) saturé par de l'eau bleue, et sur lequel tombe un grain sec.

\section{Mélanger des grains et un liquide}

Quiconque a déjà préparé une pâte à crêpe ou une sauce béchamel a été confronté à la question des grumeaux. Cet exemple, bien qu'ordinaire, d'incorporation d'un liquide, en l'occurrence le lait, à une poudre, ici la farine, permet rapidement de cerner toutes les problématiques liées à ce type de mélange. L'objectif est d'obtenir une pâte lisse et homogène, le plus rapidement possible et avec le moins d'effort.
L'apparition de grumeaux, des agrégats de farine encore secs, dispersés dans le lait, constitue le principal écueil à éviter, en ajoutant progressivement le lait et en mélangeant énergiquement.

La cuisine est loin d'être le seul domaine où des mélanges granulaires sont réalisés. Ces phénomènes d'incorporation de liquide à des matériaux dispersés sont primordiaux dans de multiples applications industrielles et nombre de phénomènes naturels. On estime par exemple que $50 \%$ des produits vendus dans le monde sont issus de 
Force d'origine capillaire des ponts liquides

Encadré 1

Assimilons dans un premier temps les grains de sable à des billes sphériques de rayon $r_{g}$. Lorsque l'on dépose un peu de liquide entre deux billes, il se crée un petit pont liquide (" pont capillaire »), qui persistera tant que sa longueur $l$ ne dépasse pas la longueur de rupture (fig. E1). Pour des grains hydrophiles, l'interface entre le liquide et l'air présente une courbure dominante tournée vers l'extérieur. Cette courbure engendre une pression de Laplace (différence de pression entre l'air et le liquide) négative, proportionnelle à la tension de surface y entre le liquide et l'air. La pression de Laplace vient donc réduire celle du liquide par rapport à la pression atmosphérique. Cette différence de pression engendre une force d'attraction entre les deux grains de sable, qui s'ajoute à la force de traction sur la ligne triple (grain/air/liquide), et les garde collés ensemble. L'amplitude de la force de cohésion résultante, due à la capillarité, s'écrit au premier ordre : $F_{\text {cap }}=2 \gamma r_{g} \cos \theta$, où $\gamma$ est de l'ordre de $70 \mathrm{mN} / \mathrm{m}$ pour l'eau, et $\theta$ est l'angle de mouillage du liquide sur le grain [4]. Pour des grains de sable de rayon $r_{g}=250 \mu \mathrm{m}$, la présence de liquide engendre une force adhésive d'amplitude de l'ordre de $100 \mu \mathrm{N}$. Le poids du grain, quant à lui, a une valeur de l'ordre du $\mu \mathrm{N}$. Ainsi, la force capillaire est une centaine de fois plus grande que la force de pesanteur, et c'est donc bien l'ajout d'une petite quantité d'eau qui permet à un château de sable de ne pas s'effondrer !

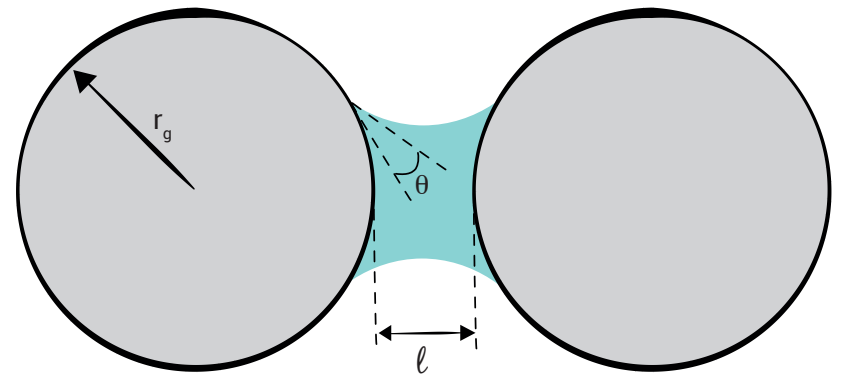

E1. Schéma d'un pont capillaire dans l'état pendulaire entre deux grains.

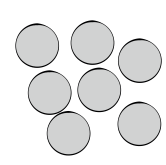

$\sec$ $0 \%$
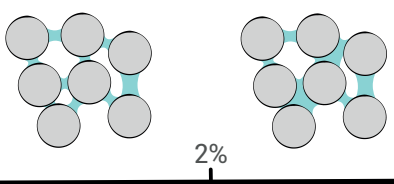

funiculaire
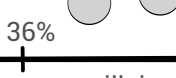

capillaire

suspension

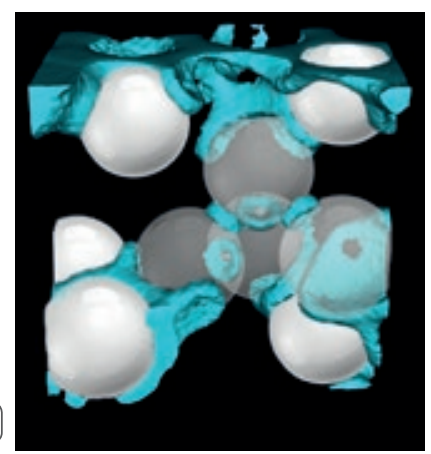

C

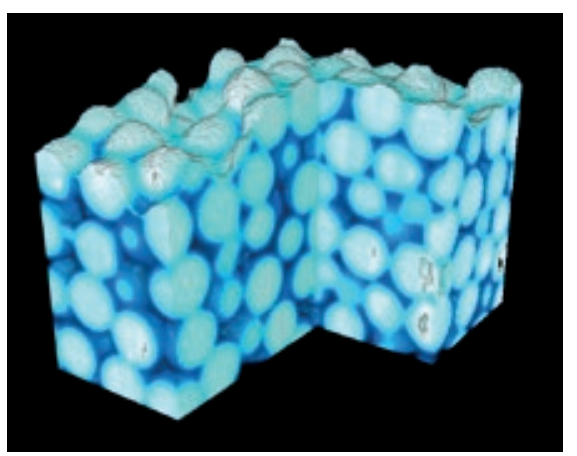

2. Les différents états d'un matériau granulaire humide statique. (a) Représentation schématique de la répartition du liquide au sein d'un matériau granulaire humide en fonction de la fraction liquide $W$. (b-c) Exemples de reconstruction de matériaux granulaires humides (billes de verre de $400 \mu \mathrm{m}$ de diamètre) obtenue par microtomographie par rayons $X$, à faible fraction liquide au début du régime funiculaire (b), puis à plus grande fraction liquide dans le régime capillaire (c). Les billes sont en blanc, le liquide en bleu.

$>>$

matières premières sous forme de grains, faisant des matériaux granulaires le second type de matériau le plus utilisé dans l'industrie après l'eau. Les principaux domaines d'activités concernés sont l'extraction minière, le génie civil, l'agro-alimentaire, les industries chimique et pharmaceutique, ainsi que l'industrie verrière, qui utilisent des matières premières ou finales granulaires [1].

Comment peut-on décrire les interactions entre des grains secs et des grains déjà humides pour mieux comprendre les mélanges entre des grains et de l'eau ? Afin de montrer les récentes avancées dans ce domaine, nous allons commencer par rappeler des éléments de la physique des milieux granulaires humides statiques, notamment les forces de cohésion entre grains. Nous pourrons alors ensuite discuter des deux cas limites : l'érosion d'un agrégat humide où des grains secs en écoulement peuvent arracher des grains collés à l'agrégat par des ponts capillaires. Puis nous illustrerons l'autre extrême où des grains secs peuvent adhérer à un agrégat humide et faire croitre sa taille. Ces deux situations illustrent la complexité de la physique des granulaires humides.

\section{Matériaux granulaires humides et forces capillaires}

Quand on parle de grains de sable humides, on pense souvent aux châteaux de sable que l'on peut réaliser étant enfant sur le bord de la plage [2]. Ces exemples de structure démontrent les effets spectaculaires de la cohésion capillaire induite par le liquide, comme illustré sur la figure 1 (p. 17). L'apport d'une faible quantité de liquide est suffisant pour changer profondément les propriétés mécaniques du matériau granulaire [3]. Ce comportement cohésif résulte de la formation de ponts liquides entre les grains (voir encadré 1).

Si les interactions capillaires sont faciles à décrire à l'échelle de deux grains, leur prise en compte dans un tas de grains est plus délicate. En effet, la répartition spatiale hétérogène du liquide modifie localement les propriétés mécaniques du matériau granulaire humide [5]. À l'échelle des grains, plusieurs états ont été historiquement définis (il y a un siècle, en 1917, par Versluys), en référence à la configuration géométrique que le liquide adopte en fonction de la fraction liquide $W$, c'est-à- 
dire le volume de liquide divisé par le volume total. Les différents états d'un matériau granulaire humide statique sont représentés sur la figure 2 a par ordre croissant de fraction liquide : l'état sec (sans liquide) est suivi de l'état pendulaire où le liquide, suspendu entre deux grains, forme des ponts capillaires indépendants. Ensuite vient l'état funiculaire (car le liquide forme des " cordons »), lorsque les ponts capillaires commencent à fusionner et le liquide relie plusieurs grains entre eux, formant ainsi des chapelets de pores complètement saturés et connectés. Puis l'état capillaire (nommé ainsi car l'eau envahit les pores des roches, "fins comme des cheveux ") intervient lorsque l'empilement granulaire est quasiment saturé (sauf en périphérie), mais reste cohésif en raison de la dépression capillaire appliquée par les ménisques liquides à l'interface liquide/air. Enfin, le milieu est sous forme de suspension lorsque les grains sont complètement immergés dans un excès de liquide. Des exemples de la morphologie d'un échantillon partiellement saturé, obtenue par microtomographie par rayons $\mathrm{X}$, sont représentés sur les figures $2 \mathrm{~b}$ et $2 \mathrm{c}$. Dans cet article nous allons considérer les deux cas limites présentant une cohésion : le régime pendulaire où le matériau peut être érodé par des grains en écoulement, et le régime capillaire où de nouveaux grains peuvent être capturés par accrétion.

La majorité des études s'est pour l'heure intéressée aux propriétés mécaniques des matériaux granulaires humides statiques, dans lesquels le liquide est réparti spatialement de manière uniforme. Néanmoins, pour réussir à percer les mystères de la transition d'un milieu granulaire sec à un
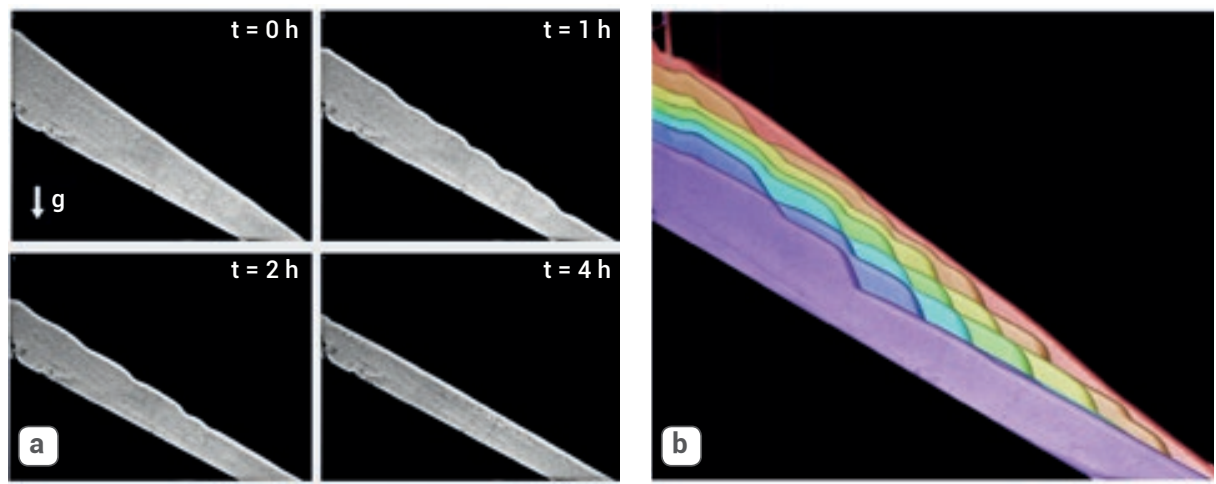

4. Expérience d'érosion à la surface pentue d'un tas bidimensionnel humide.

(a) Évolution temporelle de la morphologie d'un tas granulaire humide (aspect granuleux) sur lequel s'écoule un milieu granulaire sec (fine couche blanche) qui l'érode et induit la formation transitoire de marches (les grains sont des billes de verre de $300 \mu \mathrm{m}$ de diamètre).

(b) Superposition d'images acquises toutes les 30 minutes montrant la progression des marches vers l'amont de l'interface du tas humide sous l'effet de l'érosion [7]. Le temps va du rouge (image du fond) au violet (image au premier plan).

La largeur des images correspond à $27 \mathrm{~cm}$.

(Voir la vidéo : http://svi.cnrs.fr/spip/IMG/avi/erosion_heap.avi).

matériau humide au cours d'un mélange, il faut définir les mécanismes et la dynamique d'interaction entre des grains et du liquide au niveau des interfaces. Dans la suite, nous présentons des résultats récents liés aux deux cas limites de fraction liquide $W$, menant à l'érosion ou à l'accrétion d'un milieu granulaire humide.

\section{Érosion : casser les ponts capillaires}

Nous nous plaçons ici dans le régime pendulaire : $W<2 \%$. La question est de savoir à quelle vitesse un tel agrégat humide et cohésif s'érode lorsqu'il interagira avec un écoulement granulaire sec. Ce phénomène d'érosion intéresse particulièrement les géophysiciens qui cherchent à comprendre

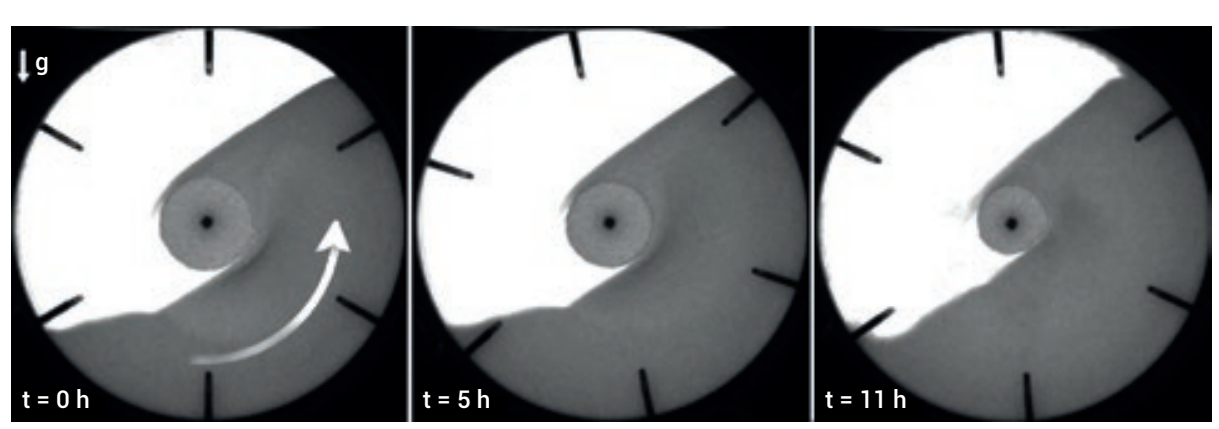

3. Exemple d'érosion d'un agrégat humide de billes de verre (disque central clair), retenu au centre d'un tambour tournant d'axe horizontal. (Diamètre des billes : $300 \mu \mathrm{m}$. Diamètre du tambour : $14 \mathrm{~cm}$. En gris foncé, le sable sec. Le vecteur g représente la gravité sur l'image de gauche). L'écoulement granulaire de surface, engendré par la rotation du tambour, arrache régulièrement des billes du pourtour de l'agrégat humide. Remarquer la diminution du diamètre de l'agrégat au cours du temps. (Une vidéo stroboscopique avec des grains plus grossiers est disponible à http://svi.cnrs.fr/spip/IMG/avi/erosion_coarse.avi) [6]. la morphologie des sols à la suite d'un éboulement ou d'une coulée pyroclastique (écoulement volcanique très violent, mélange de gaz et de particules voire de rochers, dévalant les flancs à grande vitesse). Dans son principe, l'érosion par l'écoulement granulaire ressemble grandement à l'érosion des sédiments au fond des rivières, où la force exercée par le courant sur une particule du lit permet de vaincre son poids apparent et ainsi d'éroder le fond. La principale différence, ici, réside dans la nature de la force imposée par l'écoulement sur le matériau cohésif humide.

Afin de comprendre ce mécanisme d'érosion, du matériau granulaire humide (homogène et cohésif) est tout d'abord préparé par ajout contrôlé d'un liquide (eau ou huile), puis il est soumis à un écoulement granulaire sec. Pour cela nous utilisons deux configurations. La première est un cylindre tournant d'axe horizontal, de faible épaisseur, qui permet de fixer un agrégat humide cylindrique en son centre et d'entrainer les grains secs par rotation afin qu'ils s'écoulent par-dessus l'agrégat (fig. 3). Cet écoulement granulaire arrache des grains à l'agrégat humide et ainsi l'érode. En pratique, le matériau ne s'érode que si l'énergie transférée par les chocs est suffisante pour casser les ponts capillaires et emporter le grain.

Nous avons mesuré la vitesse d'érosion des agrégats humides en fonction des paramètres du liquide [6]. Une diminution de la vitesse d'érosion est observée pour une augmentation de la fraction liquide $W$ et de la viscosité du fluide. 


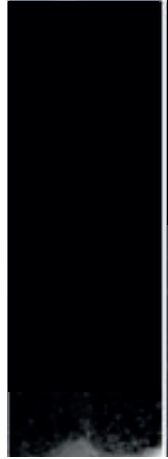

a
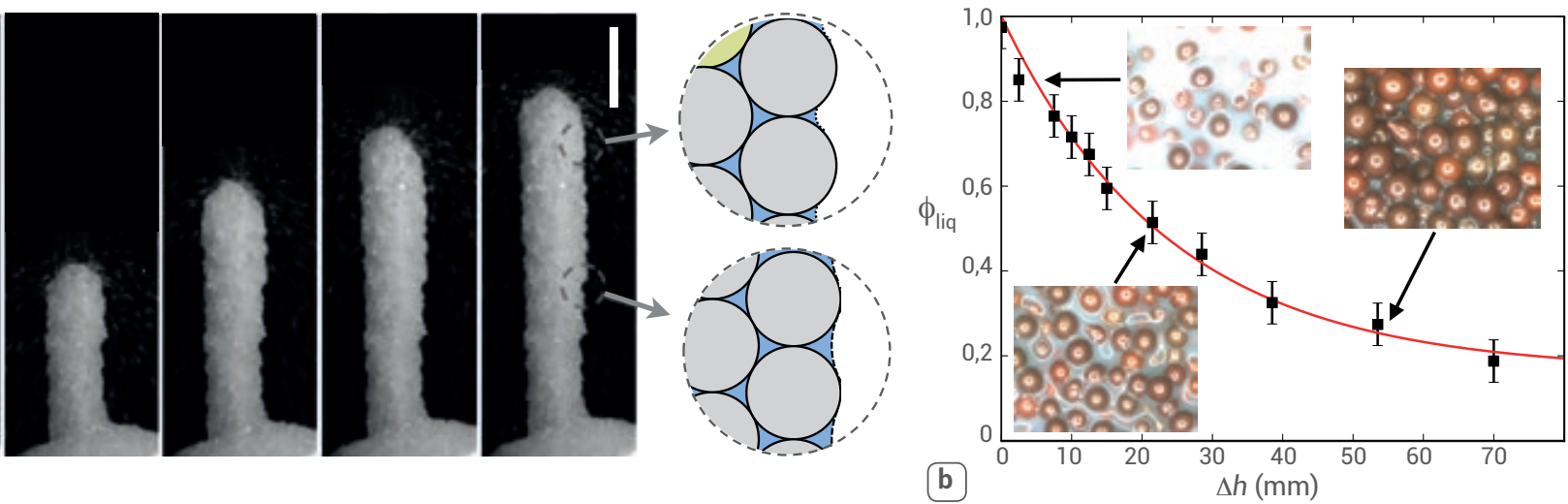

5. Croissance d'une tour granulaire sur du sable humide.

(a) Photographies de la croissance de la tour, pour des billes de verre de $340 \mu \mathrm{m}$ de diamètre et une dépression hydrostatique de $50 \mathrm{~mm}$ d'eau. La barre d'échelle est de $1 \mathrm{~cm}$. À droite : schémas illustrant la diminution du volume des ménisques capillaires extérieurs lorsqu'ils se rétractent avec la hauteur.

(b) Évolution de la fraction surfacique de liquide à la surface d'un matériau granulaire humide en fonction de la dépression imposée dans le liquide, proportionnelle à la hauteur $\Delta \mathrm{h}$ de la surface au-dessus du réservoir de liquide.
$>>$

Une seconde série d'expériences d'érosion a été réalisée à la surface pentue (plane au départ) d'un tas bidimensionnel humide (fig. 4, p. 19). Cette seconde configuration permet en effet de mieux contrôler la vitesse d'écoulement des grains secs et la et le tas humide. La vitesse d'érosion de l'interface augmente alors avec la densité des grains qui s'écoulent et avec leur vitesse. Cette expérience d'érosion a par ailleurs mis en évidence, à haute vitesse d'écoulement, une déstabilisation de la forme de l'interface initialement plane, qui module spatialement le taux d'érosion (fig. 4).

Pour interpréter ces résultats, on s'intéresse dans un premier temps à l'influence du liquide. Une fraction de liquide $W$ plus importante permet d'augmenter le volume des ponts capillaires (et leur nombre), allongeant ainsi leur longueur de rupture (voir l'encadré 1), et la viscosité module le temps nécessaire pour rompre un lien. La modélisation de l'effet des grains secs Premièrement, la densité des grains joue deux rôles : elle agit sur le poids de la couche en écoulement, donc sur la force de friction à l'interface, et elle intervient aussi dans la force d'impact, collisionnelle, des grains sur l'interface. Or, en considérant les caractéristiques moyennes de l'écoulement, l'évaluation des forces d'érosion issues de ces phénomènes donne des valeurs 10 à 100 fois inférieures à la force des ponts capillaires (voir encadré 2). pente de l'interface entre l'écoulement sec en écoulement est plus subtile.
Cette approche moyenne n'est donc pas la bonne explication : considérer l'ensemble de la distribution des valeurs des forces de contact, dont certaines dépassent la force des ponts capillaires, permet de modéliser les vitesses d'érosion. La deuxième subtilité a été révélée par l'instabilité de surface (fig. 4), analogue à la formation des cascades par les torrents. Expérimentalement, l'instabilité, tout comme la vitesse d'érosion, est plus prononcée pour les fortes pentes. Or dans ces conditions, l'épaisseur de l'écoulement, donc la pression, et la force de friction sont les plus faibles, au contraire de la vitesse des grains secs. Ce constat exclut le lien entre les forces de friction et l'érosion et souligne le rôle des forces d'impact. L'énergie cinétique des grains secs est donc la clé de ces phénomènes d'érosion [7]. Cette découverte permet ensuite de modéliser l'instabilité par l'intermédiaire de l'inertie des grains secs, qui implique que la vitesse d'érosion n'est plus en phase avec la pente locale du tas humide.

La validité de notre modèle a confirmé que l'érosion d'un agrégat humide, dans le régime pendulaire, est un phénomène probabiliste, sensible aux grandes fluctuations de forces d'impact qui sont reliées à la vitesse des grains secs en écoulement.

Voyons maintenant les mécanismes d'échanges lorsque la fraction liquide $W$ augmente fortement, en nous plaçant dans le régime capillaire.
Forces moyennes et modélisation probabiliste de l'érosion

Encadré 2

Les forces moyennes exercées par l'écoulement sur un grain humide de surface typique $\pi r_{g}^{2}$ peuvent être estimées et comparées à celle d'un pont capillaire. Pour la force frictionnelle : $F_{\text {fric }} \approx \mu \rho g h . \pi r_{g}{ }^{2} \approx 10 \mu \mathrm{N}$, où $\mu \approx 0,5$ est le coefficient de friction de l'écoulement sec sur l'interface, $\rho$ la masse volumique de la couche sèche en écoulement (1500 kg.m $\left.{ }^{-3}\right)$, $h$ l'épaisseur de la couche $(5 \mathrm{~mm})$ et $g$ la gravité. Pour la force collisionnelle provenant des impacts, la modélisation de Bagnold prédit $F_{\text {coll }} \approx \rho \dot{\gamma}^{2} r_{g}{ }^{2} \cdot \pi r_{g}{ }^{2} \approx \rho g r_{g} \cdot \pi r_{g}{ }^{2} \approx 1 \mu \mathrm{N}$, où le taux de cisaillement à l'interface $\dot{\gamma}$ est approché en ordre de grandeur par $\left(g / r_{g}\right)^{1 / 2}$. Ces deux forces moyennes sont alors inférieures à la force capillaire typique $F_{\text {cap }}$, de l'ordre de $100 \mu \mathrm{N}$. II faut donc considérer toute la distribution de probabilité des forces agissant sur l'interface (présentant une décroissance exponentielle pour les grandes forces).

La vitesse d'érosion globale résulte alors de l'intégrale de toutes les contributions des forces qui dépassent le seuil donné par la force du pont capillaire $F_{c a p}$. 

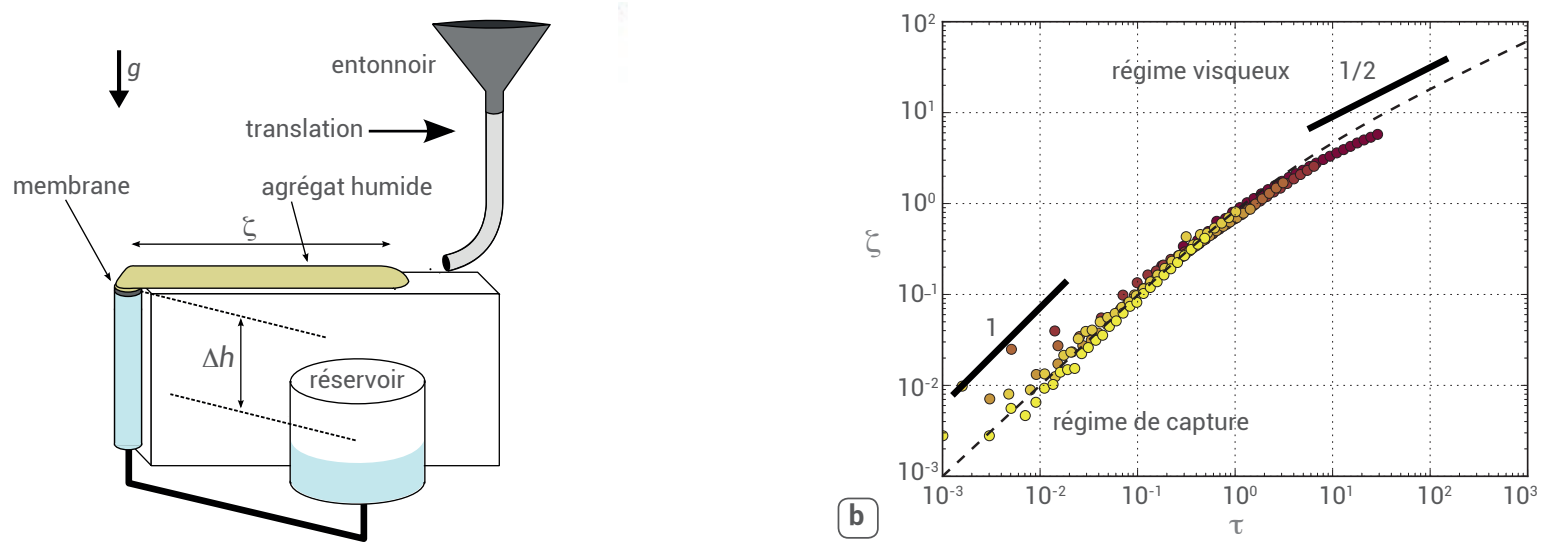

6. Croissance d'agrégats granulaires humides horizontaux.

(a) Schéma du dispositif expérimental permettant de créer des agrégats granulaires horizontaux saturés en liquide, à dépression hydrostatique $\Delta$ h contrôlée.

(b) Courbe globale prédisant l'évolution de la longueur adimensionnée $\zeta$ d'un agrégat granulaire saturé horizontal en fonction d'un temps adimensionné $\tau$. Les lois d'échelle correspondant aux deux régimes limites sont commentées dans le texte. La couleur des symboles, du foncé au clair, indique l'augmentation de la dépression hydrostatique $(\Delta \mathrm{h}=5$ à $62 \mathrm{~mm})[10]$.

\section{Accrétion : capture des grains par le liquide}

Pour visualiser l'accrétion à l'échelle du milieu granulaire, il suffit de verser du sable sec sur du sable humide. Si la quantité de liquide contenue dans le substrat humide est suffisamment importante (régime capillaire), on voit croitre une structure verticale là où un simple tas de grains conique se serait formé si le substrat avait été sec [8]. La croissance d'une tour granulaire est visible sur la figure $5 \mathrm{a}$ et montre à quel point la structure est fine et stable. Cette expérience, simple à mettre en œuvre, démontre le processus d'accrétion où de nouveaux grains viennent se coller à la structure en même temps que le liquide progresse. Le liquide peut se propager et l'agrégat peut croitre en captant des grains, on parle alors d'accrétion granulaire. Mais comment grandit une tour granulaire ? L'analyse de sa structure et de sa croissance permet d'élucider une partie des mécanismes de piégeage des grains.

Des mesures par tomographie de rayons $\mathrm{X}$ au laboratoire ont montré que l'intérieur de la tour granulaire humide est complètement saturé en liquide. Les seuls ménisques avec de l'air sont donc sur le pourtour de la colonne. Une première hypothèse est de considérer que le liquide monte dans la tour par imbibition capillaire. Ce phénomène est rencontré lorsque l'on plonge un morceau de sucre dans du café : le liquide monte spontanément dans le sucre jusqu'à une certaine hauteur (avant que le sucre ne se désagrège !). Cette situation a été décrite dans le cas de tubes capillaires au début du $\mathrm{XX}^{\mathrm{e}}$ siècle par Washburn [9], puis adaptée à des milieux poreux. Physiquement, le liquide pénètre dans les pores sous l'effet de la tension de surface et de la dépression capillaire due à la courbure de l'interface liquide/air. Pour des hauteurs assez faibles, où l'influence du poids de la colonne de liquide dans le poreux est encore négligeable, l'évolution temporelle de la hauteur de liquide ralentit proportionnellement à la racine carrée du temps à cause de la dissipation visqueuse grandissante avec la longueur traversée par le liquide.

En revanche, la dynamique de croissance de la tour suit une loi logarithmique. Ainsi, considérer uniquement l'écoulement du liquide dans le milieu poreux n'est pas suffisant pour décrire ces phénomènes d'accrétion, et il faut aussi tenir compte de la probabilité de coller un grain à l'agrégat humide lors de l'impact. Dans la tour granulaire, du fait de la dépression hydrostatique qui augmente avec la hauteur, les ménisques sur la paroi de la tour sont de plus en plus rétractés, comme schématisé à droite de la figure $5 \mathrm{a}$, et il devient donc de plus en plus difficile pour un nouveau grain d'impacter sur une zone où du liquide est présent. La fraction surfacique de liquide est donc un élément majeur pour comprendre la dynamique de croissance d'un granulaire humide. En effet, en impactant sur une poche liquide, le grain pourra être capté grâce à la dissipation visqueuse qui le freine et à la force capillaire qui le retient.

Des mesures par microscopie optique de l'aire du liquide disponible à la surface de l'agrégat ont permis de montrer que, sous l'effet de la dépression hydrostatique, la fraction surfacique de liquide $\Phi_{\text {liq }}$ diminue exponentiellement avec la hauteur, comme représenté sur la figure $5 b$, et de manière similaire à l'évolution de la probabilité de capture obtenue expérimentalement.

Afin de contrôler cette dépression hydrostatique, des expériences de croissance de tours horizontales ont été réalisées avec le dispositif expérimental présenté sur la figure 6a. L'avantage de cette configuration est que la dépression hydrostatique, et ainsi l'aire de liquide disponible pour capturer des grains, est constante pour chaque expérience effectuée à $\Delta$ h fixé. Deux régimes de croissance d'agrégats saturés, avec le temps $t$, ont alors pu être mis en évidence : (i) un régime gouverné par l'écoulement visqueux dans la structure poreuse à faible dépression (donnant une loi de croissance horizontale en $t^{1 / 2}$ ) et (ii) un régime limité par l'efficacité du processus de capture de nouveaux grains à plus grande dépression (engendrant une loi linéaire en temps car la dépression hydrostatique, donc la fraction surfacique de liquide, est constante). Toutes les dynamiques peuvent être reproduites grâce à ce double mécanisme, et l'évolution des tours horizontales est représentée sur la figure $6 b$ où les résultats sont tracés en 


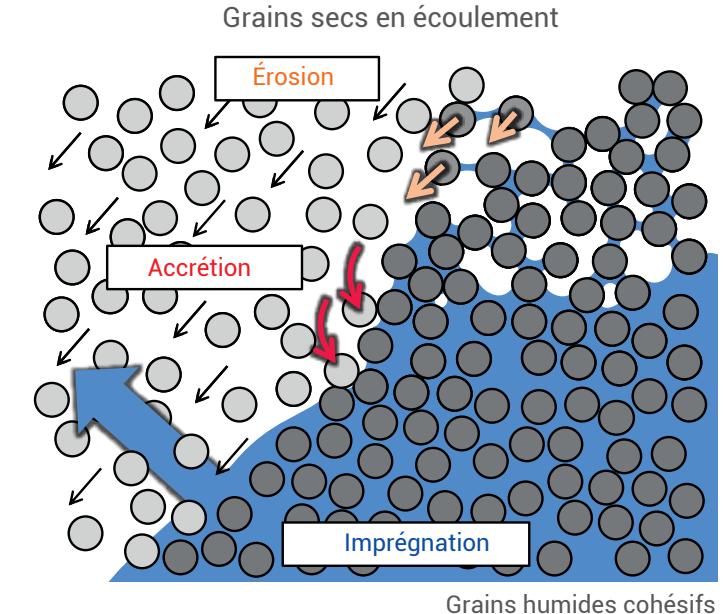

Grains humides cohésifs

. Vue synthétique des interactions entre un agrégat humide et un écoulement granulaire sec.

(a) Schéma des différents phénomènes d'échanges entre une phase granulaire sèche en écoulement et une phrase granulaire humide.

(b) Diagramme de phase qualitatif présentant les frontières attendues en fonction de l'énergie cinétique des grains secs et de la fraction liquide $W$ dans l'agrégat humide. Les points d'interrogation illustrent les zones d'ombre entourant les mécanismes exacts de la capture des grains.

$\gg>$

fonction d'une longueur $\zeta$ et d'un temps adimensionné $\tau$ avec les différents paramètres du problème, tels que les propriétés de transport liquide dans la colonne (la porosité de l'agrégat, la viscosité), le débit des grains impactant, ainsi que la valeur de la probabilité de capture à dépression nulle [10]. Bien que nous ayons identifié le principe du mécanisme, différents paramètres comme les propriétés physiques du liquide, des grains ou du jet, modifient les sources de dissipation lors de l'impact et donc la croissance d'agrégats humides de manière non triviale. Par exemple, alors que l'effet de la viscosité sur le transport du liquide est modélisé, son rôle dans la capture est ambigu. Une grande viscosité freine davantage le grain, favorisant la capture, mais rigidifie aussi la structure granulaire lors de l'impact, favorisant au contraire le rebond. Il reste ainsi à modéliser comment ces propriétés physiques modifient directement la probabilité de capturer des grains.

\section{Vers une prédiction du mélange?}

Nos études ont montré que selon la répartition de liquide contenu dans un milieu granulaire hétérogène, des grains secs en écoulement peuvent soit éroder des agrégats humides dans le régime pendulaire, soit au contraire être capturés pour de plus grandes fractions liquides (fig. 7), les faisant ainsi croitre. Il en résulte une situation typique des mélangeurs réels, décrite sur la figure 7 , où les différentes interactions de milieux granulaires humides et secs en écoulement sont présentes simultanément. L'optimisation des procédés industriels de mélange et la compréhension des phénomènes géophysiques tel que le transport éolien de sable sur sols humides nécessitent de mieux caractériser ces situations. Nous avons identifié des processus élémentaires d'échange de grains entre les phases, mais l'influence précise des propriétés du liquide ou des grains reste à comprendre (comme les influences de la viscosité ou de la densité de l'écoulement). Il faudra coupler ces effets à la déformation du matériau humide lui-même afin de pouvoir décrire toutes les étapes d'un mélange granulaire réaliste, partant d'un état initial fortement hétérogène vers une pâte granulaire finale homogène.

Ces principes décrivant l'interaction de grains secs avec des grains humides sont une première approche pour percer les mécanismes de mélange entre des grains et de l'eau tels qu'ils sont réalisés quotidiennement dans l'industrie, et apporter de nouveaux éclairages sur la physique des milieux granulaires humides. Des résultats expérimentaux modèles de ces systèmes complexes, tels que des géométries de mélanges simples avec des mélanges de grains et de liquide simples, permettront d'améliorer les approches numériques triphasiques impliquant des grains solides, une phase liquide et l'air.

\section{Références}

1 B. Andreotti, Y. Forterre et O. Pouliquen, Les milieux granulaires: Entre fluide et solide, EDP sciences (2012).

2• M. Pakpour, M. Habibi, P. Møller et D. Bonn, "How to construct the perfect sandcastle", Scientific reports (2012) 549.

$3 \cdot \mathrm{S}$. Herminghaus, "Dynamics of wet granular matter", Advances in Physics, 54 (2005) 221-261

4. P.-G. De Gennes, F. Brochard-Wyart et D. Quéré, Capillarity and Wetting Phenomena: Drops, Bubbles, Pearls, Waves, Springer (2003).

5• M. Scheel, R. Seemann, M. Brinkmann, M. Di Michiel, A. Sheppard, B. Breidenbach et $\mathrm{S}$. Herminghaus, "Morphological clues to wet granular pile stability", Nature Materials, 7 (2008) 189.

6• G. Lefebvre et P. Jop, "Erosion dynamics of a wet granular medium", Physical Review E, 88 (2013) 032205.

$7 \cdot$ G. Lefebvre, A. Merceron et P. Jop, "Interfacial Instability during Granular Erosion", Physical Review Letters, 116 (2016) 068002.

8• F. Pacheco-Vazquez, F. Moreau, N. Vandewalle et S. Dorbolo, "Sculpting sandcastles grain by grain: self-assembled sand towers", Physical Review E, 86 (2012) 051303.

9• E. W. Washburn, "The dynamics of capillary flow", Physical review, 17 (1921) 273.

10 G. Saingier, A. Sauret et P. Jop, "Accretion dynamics on wet granular materials", Physical Review Letters, 118 (2017) 208001. 